\title{
Statistical QCD with non-positive measure
}

\author{
J.C. Osborn \\ Argonne Leadership Computing Facility \\ 9700 S. Cass Avenue, Argonne, IL 60439, USA \\ Center for Computational Science, Boston University \\ Boston, MA 02215, USA \\ K. Splittorff \\ The Niels Bohr Institute \\ Blegdamsvej 17, DK-2100, Copenhagen Ø, Denmark \\ speaker, *E-mail: split@nbi.dk \\ J.J.M. Verbaarschot \\ Department of Physics and Astronomy, SUNY \\ Stony Brook, New York 11794, USA \\ E-mail: verbaarschot@cs.physics.sunysb.edu
}

\begin{abstract}
In this talk we discuss the microscopic limit of QCD at nonzero chemical potential. In this domain, where the QCD partition function is under complete analytical control, we uncover an entirely new link between the spectral density of the Dirac operator and the chiral condensate: violent complex oscillations on the microscopic scale give rise to the discontinuity of the chiral condensate at zero quark mass. We first establish this relation exactly within a random matrix framework and then analyze the importance of the individual modes by Fourier analysis.
\end{abstract}

Keywords: nonperturbative QCD, sign problem, random matrix theory

\section{Introduction}

Many firm results about QCD are based on the fact that the Euclidean partition function has a real and positive measure. Prime examples are QCD inequalities and the evaluation of the lattice QCD partition function by Monte Carlo simulations. For this reason much of the intuition gained about nonperturbative QCD is based on a probabilistic interpretation. If we introduce a chemical potential in order to favor the presence of quarks 
over anti-quarks the measure in the Euclidean partition function is no longer positive definite. In this lecture we will analyze the link between the spectral density of the QCD Dirac operator and the order parameter for spontaneous chiral symmetry breaking. We will see that the intuition based on zero chemical potential results fails completely at nonzero chemical potential.

At zero chemical potential the eigenvalues of the anti-Hermitean Dirac operator are located on the imaginary axis. The chiral condensate can be thought of as the electric field at the position on the real axis given by the quark mass, $m$, created by positive charges located at the purely imaginary eigenvalues [1]. The chiral condensate is the jump in the electric field as the quark mass passes through zero. Clearly such a jump will occur if the density of the eigenvalues/charges near the origin of the imaginary axis scales with the space-time volume [2].

At nonzero chemical potential the anti-Hermiticity of the Dirac operator is lost and the eigenvalues are located homogeneously in a strip parallel to the imaginary axis with width proportional to $\mu^{2}$ (for sufficiently large $\mu$ ). The constant of proportionality is such that, at sufficiently low temperatures, the quark mass hits the eigenvalue strip for $\mu=m_{\pi} / 2[3-5]$. From our intuition at zero chemical potential we are lead to the conclusion that at any (however small) nonzero value of the chemical potential the chiral condensate has no discontinuity when the quark mass crosses the imaginary axis. This is in sharp contrast to the expected phase diagram, see Fig. 1. (Notice however, that in phase quenched QCD the chiral condensate rotates into a pion condensate so that chiral symmetry remains broken spontaneously in spite of a vanishing chiral condensate.) Increasing the temperature tends to decrease the width of the strip of eigenvalues so that the value of the chemical potential for which the quark mass is inside the domain of the eigenvalues increases as well (see Fig. 1 for illustration).

At nonzero chemical potential the eigenvalue density of the Dirac operator is defined as

$$
\rho_{N_{f}}(x, y)=\left\langle\sum_{k} \delta\left(x-x_{k}\right) \delta\left(y-y_{k}\right)\right\rangle_{N_{f}} .
$$

Since the measure includes the fermion determinants there is no guarantee that the density is real or positive. To completely destroy the electrostatic picture it turns out that the eigenvalue density depends strongly on the quark mass $[7,8]$. Hence we can not interpret the quark mass as a test charge which, in a harmless manner, measures the chiral condensate/electric field. To study the eigenvalue density we turn to the microscopic scale of QCD [9] 


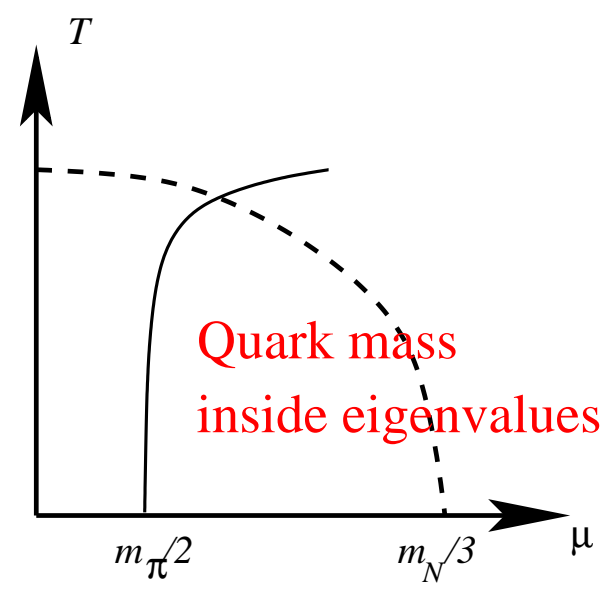

Fig. 1. Schematic phase diagram of $\mathrm{QCD}$ and region where the quark mass is inside the support of the eigenvalue density. The dashed curve indicates the chiral transition be it first order or crossover. To the right of the full line the quark mass is inside the support of the Dirac spectrum. The solid curve does not indicate a phase transition in QCD.

where the dimensionless combinations

$$
\hat{m} \equiv m \Sigma V \quad \text { and } \quad \hat{\mu}^{2} \equiv \mu^{2} F_{\pi}^{2} V
$$

are kept fixed as the volume $V$ is taken to infinity ( $\Sigma$ is the chiral condensate and $F_{\pi}$ the pion decay constant). In this limit the Compton wavelength of the pions is much larger than the linear extent of the volume and the zero momentum mode of the pions dominates the low energy effective theory of QCD $[10,11]$ resulting in a partition function that is given by a group integral. Here we will access the microscopic limit of QCD by analyzing the chiral random matrix model introduced previously [7]. We will show that the unquenched eigenvalue density is complex and oscillating when $\mu>m_{\pi} / 2$ and that these oscillations are responsible for the discontinuity of the chiral condensate [12]. The complex eigenvalue density of the Dirac operator for QCD at nonzero chemical potential was also studied for gauge fields given by a liquid of instantons [6] and strong fluctuations in the spectral density could be identified. 


\section{The random matrix model}

The random matrix partition function with $N_{f}$ quark flavors with mass $m$ and chemical potential $\mu$ is defined as [7]

$$
\mathcal{Z}_{N}^{N_{f}}(m ; \mu) \equiv \int d \Phi d \Psi w_{G}(\Phi) w_{G}(\Psi) \operatorname{det}^{N_{f}}(\mathcal{D}(\mu)+m)
$$

where the non-Hermitian Dirac operator is given by

$$
\mathcal{D}(\mu)=\left(\begin{array}{cc}
0 & i \Phi+\mu \Psi \\
i \Phi^{\dagger}+\mu \Psi^{\dagger} & 0
\end{array}\right) .
$$

The $(N+\nu) \times N$ matrices $\Phi$ and $\Psi$ are complex, $\nu$ is the topological index, and $w_{G}$ is a Gaussian weight function

$$
w_{G}(X)=\exp \left(-N \operatorname{Tr} \mathrm{X}^{\dagger} \mathrm{X}\right) .
$$

In this random matrix model, the microscopic limit is defined as the limit $N \rightarrow \infty$ where

$$
\hat{m}=2 N m \quad \text { and } \quad \hat{\mu}^{2}=2 N \mu^{2}
$$

are kept fixed. The random matrix partition function becomes identical to the microscopic QCD partition function provided that we identify, see for example [8],

$$
\begin{gathered}
\hat{m}=2 N m \rightarrow m \Sigma V, \\
\hat{\mu}^{2}=2 N \mu^{2} \rightarrow \mu^{2} F_{\pi}^{2} V .
\end{gathered}
$$

The main advantage of this matrix model as compared to the one originally introduced in [13] is that an eigenvalue representation is known [7]

$$
\mathcal{Z}_{N}^{N_{f}}(m ; \mu) \sim m^{\nu N_{f}} \int_{\mathbb{C}} \prod_{k=1}^{N} d^{2} z_{k} \mathcal{P}^{N_{f}}\left(\left\{z_{i}\right\},\left\{z_{i}^{*}\right\}, m ; \mu\right),
$$

where the joint probability distribution reads

$$
\mathcal{P}^{N_{f}}\left(\left\{z_{i}\right\},\left\{z_{i}^{*}\right\}, m ; \mu\right)=\frac{1}{\mu^{2 N}}\left|\Delta_{N}\left(\left\{z_{l}^{2}\right\}\right)\right|^{2} \prod_{k=1}^{N} w\left(z_{k}, z_{k}^{*} ; \mu\right)\left(m^{2}-z_{k}^{2}\right)^{N_{f}} .
$$

The Vandermonde determinant is defined as

$$
\Delta_{N}\left(\left\{z_{l}^{2}\right\}\right) \equiv \prod_{i>j=1}^{N}\left(z_{i}^{2}-z_{j}^{2}\right)
$$

and the weight function includes a modified Bessel function,

$$
w\left(z_{k}, z_{k}^{*} ; \mu\right)=\left|z_{k}\right|^{2 \nu+2} K_{\nu}\left(\frac{N\left(1+\mu^{2}\right)}{2 \mu^{2}}\left|z_{k}\right|^{2}\right) e^{-\frac{N\left(1-\mu^{2}\right)}{4 \mu^{2}}\left(z_{k}^{2}+z_{k}^{* 2}\right)} .
$$


Given the eigenvalue representation we can employ the powerful method of orthogonal polynomials in the complex plane developed in [14-19].

\subsection{Orthogonal polynomials, partition function and chiral condensate}

The complex orthogonal polynomials corresponding to the weight function (10) can be expressed in terms of complex Laguerre polynomials [7]

$$
p_{k}(z ; \mu)=\left(\frac{1-\mu^{2}}{N}\right)^{k} k ! L_{k}^{\nu}\left(-\frac{N z^{2}}{1-\mu^{2}}\right) .
$$

They satisfy the orthogonality relations

$$
\int_{\mathbb{C}} d^{2} z w\left(z, z^{*} ; \mu\right) p_{k}(z ; \mu) p_{l}(z ; \mu)^{*}=\delta_{k l} r_{k}^{\nu},
$$

with the norm $r_{k}^{\nu}$ given by

$$
r_{k}^{\nu}=\frac{\pi \mu^{2}\left(1+\mu^{2}\right)^{2 k+\nu} k !(k+\nu) !}{N^{2 k+2+\nu}} .
$$

The partition function for one fermion can be expressed in terms orthogonal polynomials as

$$
Z_{N}^{N_{f}=1}(m ; \mu)=m^{\nu} p_{N}(m ; \mu) .
$$

Using this partition function (14) we immediately find the chiral condensate

$$
\Sigma_{N}^{N_{f}=1}(m)=\frac{d}{d m} \log Z_{N}^{N_{f}=1}(m ; \mu)=\frac{d p_{N}(m) / d m}{p_{N}(m)}+\frac{\nu}{m} .
$$

Since we have not yet taken the microscopic limit the chiral condensate depends on $\mu$.

\section{The chiral condensate from the eigenvalue density using orthogonal polynomials}

The quenched eigenvalue density given by

$$
\rho_{N}^{N_{f}=0}\left(z, z^{*} ; \mu\right)=w\left(z, z^{*} ; \mu\right) \sum_{k=0}^{N-1} \frac{p_{k}\left(z^{*}\right) p_{k}(z)}{r_{k}} .
$$

is real and positive. The unquenched spectral density [7] which can be expressed as

$$
\rho_{N}^{N_{f}=1}\left(z, z^{*}, m ; \mu\right)=w\left(z, z^{*} ; \mu\right) \sum_{k=0}^{N-1} \frac{p_{k}\left(z^{*}\right)\left(p_{k}(z)-p_{N}(z) p_{k}(m) / p_{N}(m)\right)}{r_{k}}
$$


is not invariant under complex conjugation. The asymmetric second term causes complex valued oscillations, see figure 2 .

Here we will compute the chiral condensate (15) starting from the complex and oscillating eigenvalue density given in (17),

$$
\Sigma_{N}^{N_{f}=1}(m)=\int_{\mathbb{C}} d^{2} z \frac{2 m}{z^{2}-m^{2}} \rho_{N}^{N_{f}=1}\left(z, z^{*}, m ; \mu\right) .
$$

We will show that all integrals can be carried out using the orthogonality condition (12). The first step is to insert the above expression for the unquenched density

$$
\Sigma_{N}^{N_{f}=1}(m)=\int_{\mathbb{C}} d^{2} z \frac{2 m w}{z^{2}-m^{2}} \sum_{k=0}^{N-1} \frac{p_{k}\left(z^{*}\right)\left(p_{k}(z)-p_{N}(z) p_{k}(m) / p_{N}(m)\right)}{r_{k}}
$$

We now use the fact that

$$
\int_{\mathbb{C}} d^{2} z \frac{w\left(z, z^{*} ; \mu\right) p_{k}\left(z^{*}\right) p_{k}(z)}{z^{2}-m^{2}}=\int_{\mathbb{C}} d^{2} z \frac{w\left(z, z^{*} ; \mu\right) p_{k}\left(z^{*}\right) p_{k}(m)}{z^{2}-m^{2}}
$$

which is easily proved by first rewriting (the $c_{k}$ 's are independent of $z$ )

$$
p_{k}(z)=\left(z^{2}-m^{2}\right)\left[p_{k-1}(z)+c_{k-2} p_{k-2}(z)+\ldots\right]+p_{k}(m)
$$

and then using orthogonality, and find

$\Sigma_{N}^{N_{f}=1}(m)=\int_{\mathbb{C}} d^{2} z \frac{2 m w}{z^{2}-m^{2}} \sum_{k=0}^{N-1} \frac{p_{k}\left(z^{*}\right) p_{k}(m)}{r_{k}} \frac{1}{p_{N}(m)}\left(p_{N}(m)-p_{N}(z)\right)$

For $p_{N}(m)-p_{N}(z)$ we now insert (this is just Eq. (21) rearranged a bit)

$$
p_{N}(m)-p_{N}(z)=-\left(z^{2}-m^{2}\right)\left[p_{N-1}(z)+c_{j-2} p_{N-2}(z)+\ldots\right]
$$

and get

$$
\Sigma_{N}^{N_{f}=1}(m)=-\int_{\mathbb{C}} d^{2} z 2 m w \sum_{k=0}^{N-1} \frac{p_{k}\left(z^{*}\right) p_{k}(m)}{r_{k} p_{N}(m)}\left[p_{N-1}(z)+c_{N-2} p_{N-2}(z)+\ldots\right] .
$$

Using orthogonality we find (note that the $\mu$-dependent norms drop out)

$$
\Sigma_{N}^{N_{f}=1}(m)=\frac{2 m}{p_{N}(m)}\left[p_{N-1}(m)+c_{N-2} p_{N-2}(m)+\ldots\right] .
$$



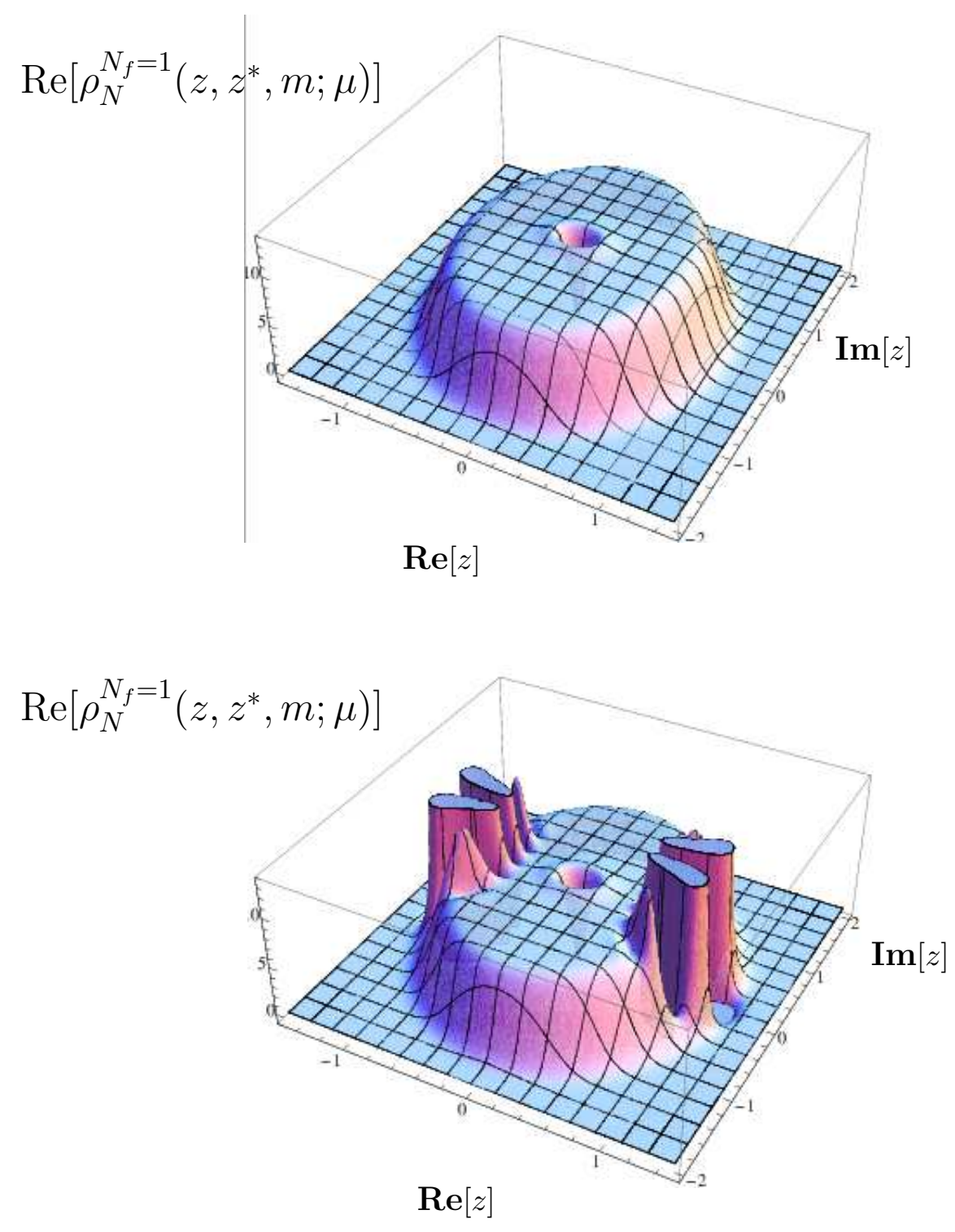

Fig. 2. The eigenvalue density of the random matrix model (3) for $N=20, \mu=0.8$ and $\nu=0$. Top: The quark mass is well outside the support of the spectrum at $m=2.0$, and the eigenvalue density is real and positive. Bottom: The quark mass is now inside the eigenvalue cloud at $m=0.6$, and oscillations starting at $z= \pm m$ extend outward. Only the real part of the spectral density is shown - the imaginary part is nonzero inside the oscillating region. 
Now,

$$
\begin{aligned}
\frac{d p_{N}(m)}{d m} & =\lim _{z \rightarrow m} \frac{p_{N}(z)-p_{N}(m)}{z-m} \\
& =\lim _{z \rightarrow m} 2 m \frac{p_{N}(z)-p_{N}(m)}{z^{2}-m^{2}} \\
& =\lim _{z \rightarrow m} 2 m\left[p_{N-1}(z)+c_{N-2} p_{N-2}(z)+\ldots\right] \\
& =2 m\left[p_{N-1}(m)+c_{N-2} p_{N-2}(m)+\ldots\right],
\end{aligned}
$$

so that

$$
\Sigma_{N}^{N_{f}=1}(m)=\frac{p_{N}^{\prime}(m)}{p_{N}(m)} .
$$

This is the desired relation (15) up to the term $\nu / m$ which is due to the contribution of $\nu$ exact zero modes that were not included in the spectral density. The result of this section originally appeared in [12].

Note that the proof relies entirely on orthogonality properties of polynomials. The detailed cancellations leading to the simple form of the chiral condensate are in this way linked to the orthogonality properties in the complex plane.

The above proof was given for finite $N$ and is of course also valid in the universal microscopic limit. As shown in [12], the proof can also be carried through in the microscopic limit without relying on the finite $N$ chiral random matrix model. That is, one can start from the microscopic limit of the spectral density (17), which is $[7,8]$

$$
\begin{aligned}
& \rho_{N_{f}=1}^{(\nu)}\left(\hat{z}, \hat{z}^{*}, \hat{m} ; \hat{\mu}\right)=\frac{|\hat{z}|^{2}}{2 \pi \hat{\mu}^{2}} K_{\nu}\left(\frac{|\hat{z}|^{2}}{4 \hat{\mu}^{2}}\right) \mathrm{e}^{-\frac{\hat{z}^{2}+\hat{z}^{* 2}}{8 \hat{\mu}^{2}}} \\
& \times\left(\int_{0}^{1} d t t \mathrm{e}^{-2 \hat{\mu}^{2} t^{2}} I_{\nu}(\hat{z} t) I_{\nu}\left(\hat{z}^{*} t\right)-\frac{I_{\nu}(\hat{z})}{I_{\nu}(\hat{m})} \int_{0}^{1} d t t \mathrm{e}^{-2 \hat{\mu}^{2} t^{2}} I_{\nu}(\hat{m} t) I_{\nu}\left(\hat{z}^{*} t\right)\right),
\end{aligned}
$$

and compute the chiral condensate using complex contour integration techniques. In the microscopic limit the cancellations are even more dramatic than at finite $N$. The entire $\mu$ dependence of the eigenvalue density vanishes upon integration and leaves us with a $\mu$ independent chiral condensate

$$
\Sigma(\hat{m})=\frac{I_{\nu}^{\prime}(\hat{m})}{I_{\nu}(\hat{m})} .
$$

Rather than repeating the proof of [12] we now focus on the Fourier components of the eigenvalue density that are responsible for chiral symmetry breaking. 
Note that the first term in (28) is the quenched eigenvalue density [20] and the effect of unquenching is in the second term. This motivates the notation

$$
\rho_{N_{f}=1}^{(\nu)}=\rho_{Q}^{(\nu)}-\rho_{U}^{(\nu)}
$$

which we shall make use of below.

\section{The Fourier transform of the eigenvalue density}

To further expose the nature of the cancellations leading to a $\mu$ independent chiral condensate we now consider the Fourier transform of the eigenvalue density. The motivation to do so is that the discontinuity in the chiral condensate is due to the strongly oscillating part of the spectral density [12]. To evaluate the chiral condensate from the eigenvalue density

$$
\Sigma_{N_{f}}(m)=\int d x d y \frac{\rho_{N_{f}}(x, y)}{x+i y-m}
$$

illustrates the effects of the phase of the fermion determinant in QCD at nonzero chemical potential. In particular for $\mu>m_{\pi} / 2$ where the sign problem is acute [21], it would be desirable if we could find a real and positive reformulation of this integral.

The Fourier transform of the eigenvalue density along the imaginary axis is defined by

$$
\begin{aligned}
\tilde{\rho}_{N_{f}}(x, t) & \equiv \int_{-\infty}^{\infty} d y e^{-i y t} \rho_{N_{f}}(x, y) \\
& =\left\langle\sum_{k} \delta\left(x-x_{k}\right) e^{-i y_{k} t}\right\rangle_{N_{f}} .
\end{aligned}
$$

Since we integrate over all $y$ the Fourier transform is necessarily real.

The chiral condensate becomes

$$
\begin{aligned}
\Sigma(m) & =\int d x d y \frac{\rho(x, y)}{x+i y-m}, \\
& =\int d x d y \frac{1}{x+i y-m} \frac{1}{2 \pi} \int d t e^{i y t} \tilde{\rho}(x, t), \\
& =\frac{1}{2 \pi} \int d x \int d t \tilde{\rho}(x, t) \int d y \frac{e^{i y t}}{x+i y-m}, \\
& =\int d x \int d t \tilde{\rho}(x, t) e^{t(m-x)} \theta(x-m) .
\end{aligned}
$$

Now the integrand is real and positive if $\tilde{\rho}$ is. Below we will see that this is the case for the asymptotic limit of the unquenched microscopic density. 


\subsection{The asymptotic limit of the microscopic spectral density}

In the limit where the microscopic variables $\hat{m}$ and $\hat{\mu}$ are much larger than unity, the expression for the eigenvalue density simplifies considerably. The quenched part of the microscopic eigenvalue distribution is simply constant and equal to $1 / 4 \hat{\mu}^{2}$ for $\operatorname{Re}[\hat{z}]<2 \hat{\mu}^{2}$. This leads to a chiral condensate that decreases linearly with the quark mass

$$
\Sigma_{Q}(\hat{m})=\frac{\hat{m}}{2 \hat{\mu}^{2}} \quad \text { for } \quad|\hat{m}|<2 \hat{\mu}^{2} .
$$

In the asymptotic limit the oscillating part of the microscopic eigenvalue density reads [12] (here and below $\hat{m} \geq 0$ )

$$
\rho_{U}(\hat{x}, \hat{y})=\frac{1}{4 \pi \hat{\mu}^{2}} e^{(\hat{m}-\hat{x}-i \hat{y})\left(\hat{m}+3 \hat{x}-8 \hat{\mu}^{2}-i \hat{y}\right) /\left(8 \hat{\mu}^{2}\right)} .
$$

Obviously the amplitude grows exponentially with the volume whereas the period is inversely proportional to the volume.

By simple Gaussian integration it follows that the Fourier transform of $\rho_{U}$ is

$$
\tilde{\rho}_{U}(\hat{x}, \hat{t})=\frac{1}{\sqrt{2 \pi \hat{\mu}^{2}}} e^{-2 \hat{\mu}^{2}(\hat{t}-1)^{2}-\hat{x}^{2} / 2 \hat{\mu}^{2}-\hat{m} \hat{t}-(\hat{t}-2) \hat{x}+2 \hat{m}} .
$$

It is a positive definite and nicely behaved function with maximum at $\hat{t}=$ $1-(\hat{m}+\hat{x}) / 4 \hat{\mu}^{2}$ (see Fig. 3). The contribution to the chiral condensate of the unquenched part of the spectral density thus becomes an integral over a positive definite function of the real part of the eigenvalue, $x$, and the Fourier coordinate, $t$,

$$
\Sigma_{U}(\hat{m})=\frac{1}{\sqrt{2 \pi \hat{\mu}^{2}}} \int_{-2 \hat{\mu}^{2}}^{2 \hat{\mu}^{2}} d \hat{x} \int_{-\infty}^{\infty} d \hat{t} e^{-2 \hat{\mu}^{2}(\hat{t}-1)^{2}-\hat{x}^{2} / 2 \hat{\mu}^{2}-2(\hat{t}-1) \hat{x}} \theta(\hat{x}-\hat{m})(37)
$$

A simple computation yields

$$
\begin{aligned}
\Sigma_{U}(\hat{m}) & =\frac{1}{\sqrt{2 \pi \hat{\mu}^{2}}} \int_{-2 \hat{\mu}^{2}}^{2 \hat{\mu}^{2}} d \hat{x} \theta(\hat{x}-\hat{m}) e^{-\hat{x}^{2} / 2 \hat{\mu}^{2}} \int_{-\infty}^{\infty} d \hat{q} e^{-2 \hat{\mu}^{2} \hat{q}^{2}-2 \hat{q} \hat{x}} \\
& =\frac{1}{\sqrt{2 \pi \hat{\mu}^{2}}} \int_{-2 \hat{\mu}^{2}}^{2 \hat{\mu}^{2}} d \hat{x} \theta(\hat{x}-\hat{m}) e^{-\hat{x}^{2} / 2 \hat{\mu}^{2}} \sqrt{\frac{\pi}{2 \hat{\mu}^{2}}} e^{\hat{x}^{2} / 2 \hat{\mu}^{2}} \\
& =\frac{1}{2 \hat{\mu}^{2}} \int_{-2 \hat{\mu}^{2}}^{2 \hat{\mu}^{2}} d \hat{x} \theta(\hat{x}-\hat{m}), \\
& =1-\frac{m}{2 \hat{\mu}^{2}}
\end{aligned}
$$



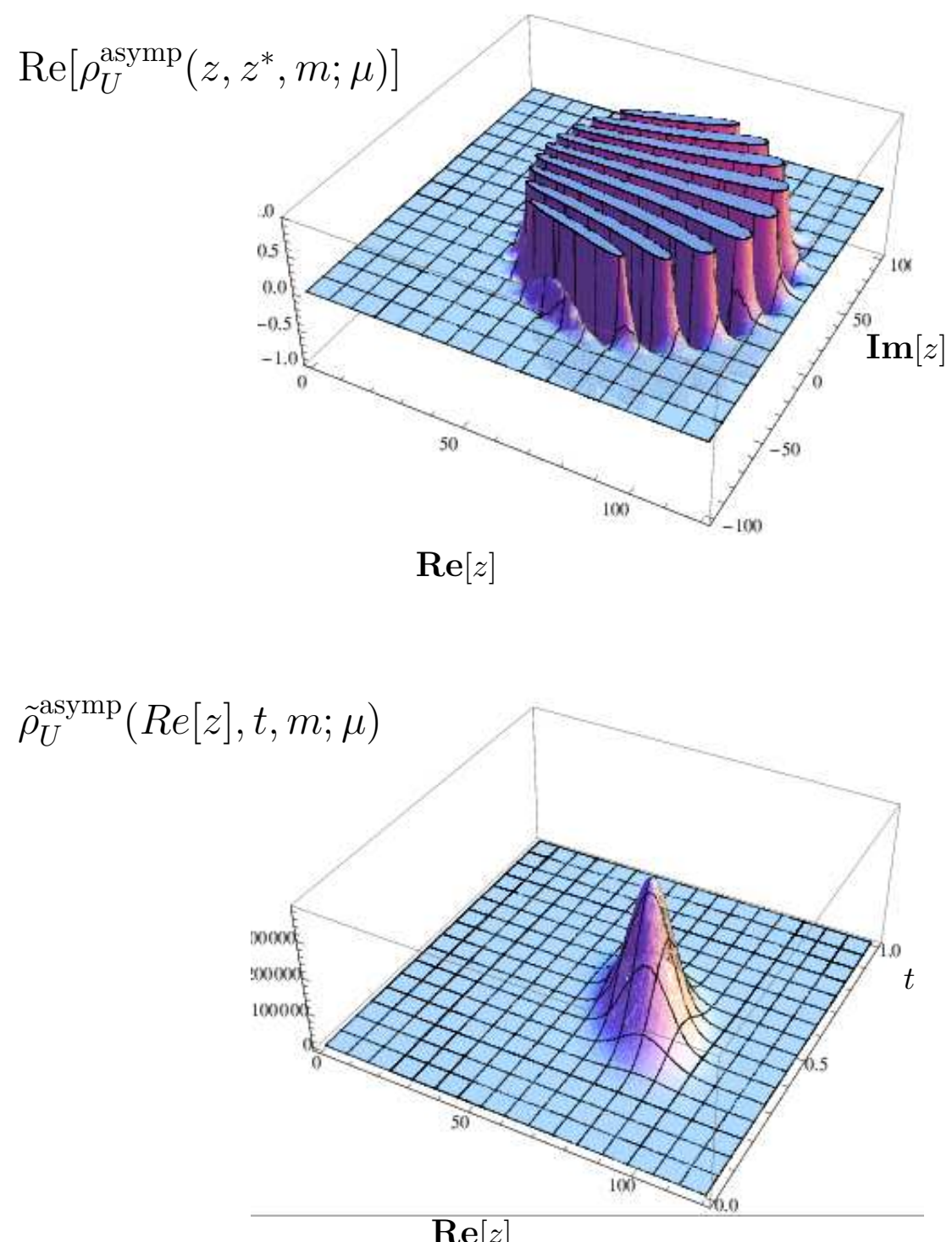

Fig. 3. Top: The asymptotic limit of the oscillating part of the unquenched eigenvalue density. Bottom: The Fourier transform of the asymptotic limit of the oscillating part of the unquenched eigenvalue density. In both cases $\hat{m}=30$ and $\hat{\mu}=7$.

The full chiral condensate is obtained by adding the quenched part (34), note that the $\mu$ dependent term drops out. The message to take away from 
this derivation is that the calculation of the chiral condensate may simplify by using the mixed Fourier transform of the spectral density introduced above.

\section{Conclusions}

Chiral symmetry breaking has been linked to complex oscillations on the microscopic scale. The cancellation of these violent oscillations, which result in the correct value of the chiral condensate, demonstrates the numerical difficulties in dealing with the sign problem when the quark mass is inside the support of the eigenvalues. At low temperatures this occurs for $\mu>m_{\pi} / 2$. We have shown that the mechanism of these cancellations can be understood in terms of orthogonality relations for the orthogonal polynomials corresponding to a random matrix theory that describes the microscopic limit of QCD. The derivation is even valid for finite size random matrices. The fact that the oscillations predominantly occur parallel to the imaginary axis motivated us to study the Fourier transform of the eigenvalue density in the imaginary part of the eigenvalue. In the asymptotic limit the resulting Fourier transformed eigenvalue density is real and positive, and in this formulation a probabilistic interpretation of the contributions to the chiral condensate is possible.

It would be most interesting to generalize these results to the $p$-domain of chiral perturbation theory.

\section{Acknowledgments}

It is a pleasure to thank the FTPI faculty and staff for an exciting workshop and Poul Henrik Damgaard for stimulating discussions. This work was supported by U.S. DOE Grant No. DE-FG-88ER40388 (JV), the Villum Kann Rassmussen Foundation (JV), the Danish National Bank (JV) and the Danish Natural Science Research Council (KS).

\section{References}

1. I. Barbour, N. E. Behilil, E. Dagotto, F. Karsch, A. Moreo, M. Stone and H. W. Wyld, Nucl. Phys. B 275, 296 (1986).

2. T. Banks and A. Casher, Nucl. Phys. B169, 103 (1980).

3. P. E. Gibbs, Glasgow University preprint PRINT-86-0389 (1986) unpublished.

4. D. Toublan and J. J. M. Verbaarschot, Int. J. Mod. Phys. B 15, 1404 (2001) [hep-th/0001110].

5. M. P. Lombardo, J. B. Kogut and D. K. Sinclair, Phys. Rev. D 54 (1996) 2303 [arXiv:hep-lat/9511026]. 
6. T. Schafer, Phys. Rev. D 57, 3950 (1998) [arXiv:hep-ph/9708256].

7. J. C. Osborn, Phys. Rev. Lett. 93, 222001 (2004), [arXiv:hep-th/0403131].

8. G. Akemann, J. C. Osborn, K. Splittorff and J. J. M. Verbaarschot, Nucl. Phys. B 712, 287 (2005), arXiv:hep-th/0411030.

9. E. V. Shuryak and J. J. M. Verbaarschot, Nucl. Phys. A 560, 306 (1993) [arXiv:hep-th/9212088].

10. J. Gasser and H. Leutwyler, Phys. Lett. B 184, 83 (1987).

11. H. Leutwyler and A. Smilga, Phys. Rev. D 46, 5607 (1992).

12. J. C. Osborn, K. Splittorff and J. J. M. Verbaarschot, Phys. Rev. Lett. 94, 202001 (2005), [arXiv:hep-th/0501210]; arXiv:0805.1303 [hep-th].

13. M.A. Stephanov, Phys. Rev. Lett. 76 (1996) 4472 [arXiv:hep-lat/9604003].

14. Y.V. Fyodorov and H.-J Sommers, J.Phys.A:Math.Gen. 36 (2003), 3303 [arXiv:nlin/0207051].

15. G. Akemann and G. Vernizzi, Nucl. Phys. B 660 (2003) 532 [hep-th/0212051].

16. G. Akemann, Acta Phys. Polon. B 34 (2003) 4653 [hep-th/0307116].

17. M.C. Bergère, [hep-th/0311227].

18. M.C. Bergère, [hep-th/0404126].

19. G. Akemann and A. Pottier, J. Phys. A 37, L453 (2004) [math-ph/0404068].

20. K. Splittorff and J. J. M. Verbaarschot, Nucl. Phys. B 683, 467 (2004) [arXiv:hep-th/0310271].

21. K. Splittorff, PoS LAT2006, 023 (2006) [arXiv:hep-lat/0610072]; K. Splittorff and J. J. M. Verbaarschot, Phys. Rev. Lett. 98, 031601 (2007) [arXiv:heplat/0609076]; Phys. Rev. D 75, 116003 (2007) [arXiv:hep-lat/0702011]. 